Retromolar tracheal tube positioning for patients undergoing faciomaxillary surgery

\section{To the Editor:}

In patients undergoing complex faciomaxillary surgery, orotracheal [need for inter-maxillary fixation (IMF)] and nasotracheal intubations (nasal injury, skull base fracture, rhinoplasty) may be unsuitable. ${ }^{1}$ Alternative options include: short-term tracheostomy (invasive, scar) and submental intubation (orocutaneous fistula $)^{2}$ but have their own limitations.

A closer observation by the author (S.S.S.) revealed that there is enough space in the retromolar region for an endotracheal tube (ETT) to be placed and allow IMF without tube compression or kinking. We successfully utilized tracheal tube positioning in the retromolar trigone ${ }^{3}$ for short-term intraoperative use in 42 adult patients undergoing a variety of maxillofacial procedures.

After routine preoperative preparation (orodental examination, informed consent, aspiration prophylaxis, IM glycopyrrolate), anesthetic induction, skeletal muscle relaxation and orotracheal intubation with a reinforced tracheal tube are carried out in a standardized manner. While instituting IMF, the ETT is moved laterally along the buccal sulcus beyond the last molar and placed in the retromolar groove to be taken out at the angle of the mouth (Figure). On completion of the scheduled procedure, the IMF is cut open

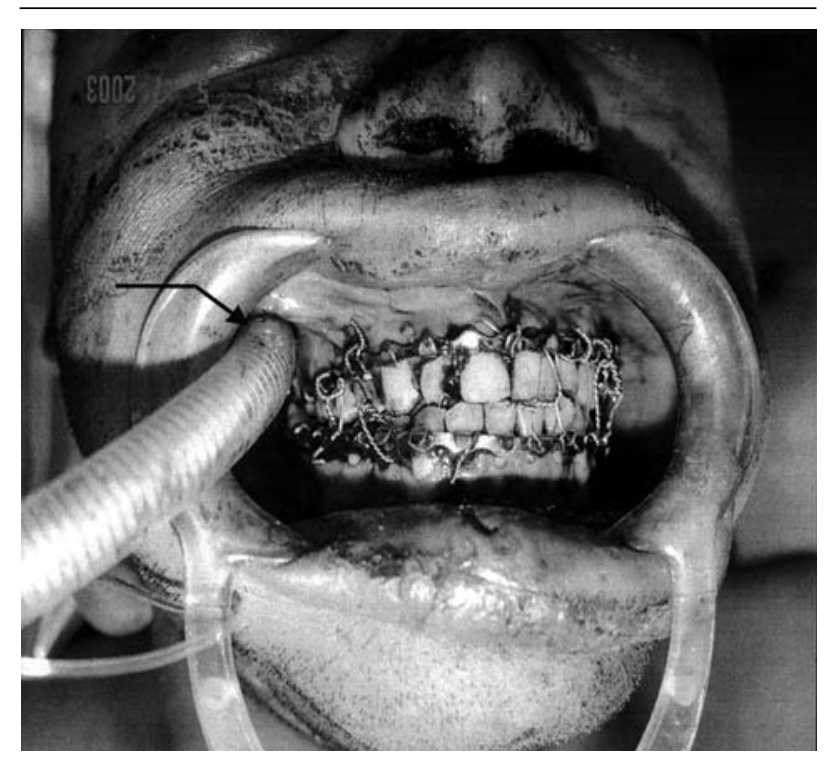

FIGURE Retromolar placement of the tracheal tube with intermaxillary wire fixation in place. and usual orotracheal tube position is resumed. Adequacy of oxygenation, ventilation and tube patency (peak airway pressure) are monitored continuously.

"Retromolar" tracheal tube positioning offers the following advantages: it is noninvasive, easy to perform, non-traumatic and time efficient; tube patency can be easily monitored, and most importantly, an optimal intraoperative control of dental occlusion can be achieved. Though we did not encounter any difficulty or complication, the potential disadvantages could be: limited space, adjoining soft tissue and mucosal trauma, long buccal nerve palsy (loss of sensation on buccal mucosa), inadequate/difficult occlusion and accidental extubation. Furthermore, its applicability in cases with pre-existing temporomandibular pathology and efficacy for long-term postoperative use is questionable.

In conclusion, retromolar positioning of the tracheal tube is an attractive option for maintaining the airway in patients during faciomaxillary procedures.

Amitabh Dutta MD

Vivek Kumar MS

S. S. Saha MS MCH

Jayashree Sood MD FFARCS

R. K. Khazanchi MS MCH

Sir Ganga Ram Hospital, New Delhi, India

E-mail: duttaamitabh@yahoo.co.in

\section{References}

1 Zmyslowski WP, Maloney PL. Nasotracheal intubation in the presence of facial fractures (Letter). JAMA 1989; 262: 1327-8.

2 Hernandez Altemir FH. Nasotracheal intubation in patients with facial fractures (Letter). Plast Reconstr Surg 1992; 89: 165-6.

3 Sharma PK, Schuller DE, Baker SR. Malignant neoplasms of the oral cavity. In: Cummings CW, Fredrickson JM, Harker LA, Krause CJ, Schuller DE, Richardson MA (Eds). Otolarngology Head \& Neck Surgery, 3rd ed. St. Louis: Mosby; 1998: 1422.

\section{Mainstem bronchial diameter for the left-sided Broncho-Cath® double- lumen tube: an in vitro study}

\section{To the Editor:}

Adequacy of a double-lumen tube (DLT) size has been determined depending on whether the bronchial cuff volume required for isolation is $>$ zero but $\leq$ the resting volume. ${ }^{1,2}$ Recently, direct measurement of the left 